\title{
Progressive hemiparesis in a 75-year-old man
}

\section{Peter M Fernandes, ${ }^{1}$ Martin R Turner, ${ }^{2}$ Martin Zeidler, ${ }^{3}$ Colin Smith, ${ }^{4}$ Richard Davenport ${ }^{1}$}

\begin{abstract}
${ }^{1}$ Department of Clinical Neurosciences, Western General Hospital, Edinburgh, UK

${ }^{2}$ Nuffield Department of Clinical Neurosciences, John Radcliffe Hospital, Oxford University, Oxford, UK

${ }^{3}$ Victoria Hospital, Kirkcaldy, UK ${ }^{4}$ Department of Neuropathology, Western General Hospital, Edinburgh, UK

Correspondence to Dr Peter M Fernandes, Department of Clinical Neurosciences, Western General Hospital, Edinburgh, UK; peterfernandes@nhs.net
\end{abstract}

Accepted 2 September 2014 Published Online First

24 September 2014

\section{CrossMark}

To cite: Fernandes PM, Turner MR, Zeidler $M$, et al. Pract Neurol 2015;15:63-71.

\section{HISTORY}

A 75-year-old, right-handed, married, retired dockyard electrical engineer presented in 2005 with a 35-year history of progressive right leg weakness, such that he was finding walking difficult. Over the 10 years before presentation, he had also developed right hand weakness, with loss of feeling in the right ring and little fingers. He had difficulty writing, using cutlery, washing and dressing. Aside from approximately $10 \mathrm{~kg}$ of weight loss over several months there were no other symptoms; in particular, there were no bulbar, cognitive or autonomic symptoms and no neck pain.

His past history included atrial fibrillation and supraventricular tachycardia, mitral valve prolapse, left ventricular failure and an atrioseptal defect repaired in 1990. A pacemaker was inserted for supraventricular tachycardia in 1990. Pleural plaques were identified in 1990, thought to be secondary to previous asbestos exposure. His medications at presentation were digoxin, ramipril, verapamil, furosemide, warfarin and amiodarone. He had a normal developmental history and had no difficulties with sports or other physical activities when younger. He did not drink alcohol to excess and was an ex-smoker, with a 10 -year pack history. His parents had died of strokes (his mother aged 78 years and father aged 66 years), but there was no other family history.

\section{EXAMINATION}

He looked well, and was comfortable at rest. There were no cranial nerves abnormalities. There was wasting of the intrinsic hand muscles bilaterally, right biceps and right forearm. The right calf was thinner than the left. There were no fasciculations. Tone was normal. There was weakness of right finger extension, abduction and thumb abduction, but finger flexion and proximal strength were normal. Hip flexion on the right was weak, and he could not lift his leg off the couch. The remaining right leg flexors were weak but the extensors were normal. Strength in the left arm and leg was normal. Deep tendon reflexes were present, apart from the ankle jerks. The right knee jerk was brisker than the left. Plantar responses were extensor. Sensory examination was normal to light touch or pinprick sensation. There was absent vibration sense distal to the costal margin and, in the arms, distal to the elbows bilaterally. Joint position sense was decreased in the right great toe, but intact elsewhere. He had a hemiparetic gait. Romberg's test was negative.

\section{CLINICAL PROGRESS}

Box 1 shows the patient's investigation results at presentation (in 2005). No diagnosis was made and he was reviewed in January 2006. There was no change in clinical condition at this time. Cerebrospinal fluid (CSF) examination was discussed with him, but he did not wish to stop his warfarin.

He was seen again in June 2006 and January 2007, with modest progression of his right-sided wasting and weakness. He could still walk but found a wheelchair helpful. He was discharged from the neurology clinic in 2007 with a clinical diagnosis. Over the next few years his weakness progressed gradually, but remained restricted to the right side. He continued to lose weight despite a good appetite.

During 2010, he had repeated admissions to hospital with dyspnoea, thought to be secondary to left ventricular failure. This was difficult to treat, given coexistent low blood pressure and renal impairment. He also developed swallowing difficulties, with frequent coughing after swallowing. He was admitted in November 2010 with worsening dyspnoea, orthopnoea and 


\section{Box 1 Investigations}

\section{Bloods}

- Normal or negative:

- Vitamin B12, folate, full blood count, plasma viscosity, urea and electrolytes, liver function tests, glucose, plasma viscosity, protein electrophoresis, cholesterol, syphilis serology, antinuclear factor, thyroid stimulating hormone, very long chain fatty acids.

- Abnormal:

- Lymphocyte count 0.68 (1.5-4.8).

- $\gamma$-glutamyl transferase $181(0-50)$.

- Triglycerides 0.7 (0.85-2.0).

- Free thyroxine 21 (10-19).

\section{Radiology}

- CT scan of head (2005): mild/moderate generalised atrophy, most marked within the cerebellum. Diffuse white matter low attenuation likely secondary to small vessel change.

- CT scan of cervical spine (2005): no evidence of cord compression or significant neural exit foraminal stenoses, although degenerative osteophytes at $\mathrm{C} 5 / 6$ and C6/7. Cervical scoliosis to the right. (MR scanning contraindicated due to pacemaker).

- CT chest (2010): emphysema, extensive pleural plaques, interstitial changes in keeping with pulmonary oedema.

\section{Neurophysiology}

- Nerve conduction studies and electromyography (2005): minor changes only, felt to be of no clinical relevance.

- Visual evoked potentials (2006): normal.

\section{Other investigations}

- Spirometry (2010): FEV1 0.9 (27\% of predicted value); FVC 1.61 (36\% of predicted value); FEV1/FVC ratio $56 \%$ (predicted value $77 \%$ ).

- Echocardiogram (2010): severe mitral regurgitation, grossly dilated left atrium, dilated right heart, mild/ moderate pulmonary hypertension, good left ventricular systolic function.

declining exercise tolerance. While in hospital, he developed a productive cough and type 2 respiratory failure, with probable aspiration pneumonia. He deteriorated despite treatment and died in hospital in December 2010.

A postmortem examination confirmed bronchopneumonia as the cause of death.

\section{DR MARTIN TURNER'S DISCUSSION}

From the history, the information may be reduced to essentials as follows:
- Time course (in years): onset at 40 , presented at 75 , death at 80 .

- Primary symptom: what is the main problem reported (or sometimes, not reported) by the patient? In this case, the key symptom was right leg weakness rather than sensory loss.

- Onset: his symptoms developed gradually.

- Progression: he had progressive hemiparesis worsening over 35 years.

- Central versus peripheral lesion: in the vast majority of neurology cases, it is one or the other. In this case, there is evidence of both!

In a similar fashion, the examination can be reduced to a few salient points. He had a right hemiparesis, with some upper motor neurone signs: pyramidal weakness, brisk right knee jerk and extensor toes. However, the tone was normal and the right calf was thinned, with wasted hand intrinsic muscles-but without fasciculation. In addition, there was a bilateral loss of vibration sense, with normal proprioception. We are told that the patient was 'normal above the neck', but I would like to know specifically about neck weakness; was there the 'dropped head syndrome' found in a number of neuromuscular conditions, including amyotrophic lateral sclerosis (ALS, otherwise known as motor neurone disease, MND); and what of the presence or absence of facial reflexes (in particular, jaw and glabellar reflexes)? There is no mention of neuropsychiatric disturbance, including emotionality, a common symptom in MND with corticobulbar involvement, though the chronology of his last year of life suggests late bulbar dysfunction, with aspiration pneumonia as the cause of death.

The list of investigations includes some abnormal results, which may be relevant. The CT head and cervical spine showed cerebellar atrophy and, importantly, indicated no abnormalities with the bones of the neck. A slight lymphopenia was noted, but he was not anaemic. The triglycerides were slightly low. The $\gamma$-glutamyl transferase was three times the upper limit of normal-we are told that the patient did not drink alcohol to excess, but establishing a precise alcohol intake is necessary. The neurophysiology was noncontributory in diagnostic terms in this case.

There are several possible 'distracters' thrown in to make my task more difficult. The pacemaker prevented MR scanning of the brain or cervical spinal cord, which would exclude structural causes or provide clues to alternative aetiologies. Similarly, a lumbar puncture was precluded by anticoagulation with warfarin. The pleural plaques indicate asbestos exposure and raise the possibility of asbestosis (which is associated with peripheral neuropathy) although asbestosis is not specifically mentioned in the clinical history or the report of the CT scan of the chest. He was on amiodarone, which can cause a peripheral neuropathy. He had numbness of the medial two fingers of the right hand -perhaps, an incidental right ulnar neuropathy in addition to his other, main, diagnosis? 


\section{Nutritional neurology}

There are a number of disorders that can manifest as neurological signs or symptoms, caused by inbuilt errors of metabolism or dietary deficiencies (or both). Functional vitamin B12 deficiency may cause subacute combined degeneration of the spinal cord despite normal B12 levels in the blood. This extremely rare disorder is caused by faulty metabolism of B12 (such as failure of intracellular B12 transport by transcobalamin- $2^{1}$ ) and can be confirmed by investigating for raised levels of methylmalonic acid or homocysteine.

I have been previously involved with a case of progressive hemiparesis, presenting in a 44-year-old man. ${ }^{2}$ An MRI brain showed corticospinal tract hyperintensity on the fluid attenuation inversion recovery sequences. This is found in about $40 \%$ of cases of MND although it is not specific. ${ }^{3}$ Neurophysiology, in this case, showed electromyography (EMG) evidence of extensive global denervation with normal nerve conduction. Lumbar puncture was normal, and antinuclear antibody/extractable nuclear antigen/antineuronal antibody screens were all negative. Blood tests showed anaemia with low mean cell volume, iron and folate, prompting a screen for incidental coeliac disease. Antiendomysial antibodies were positive, and a duodenal biopsy was typical for coeliac disease. The patient improved slowly after commencing a glutenfree diet, with the conclusion that the neurological dysfunction was associated with coeliac disease. The clinical spectrum of neurological dysfunction in coeliac disease is broad ${ }^{4}$ (box 2).

At this point, I will make a personal observation about the ubiquitous testing of folate in neurology. I am not aware of any independent primarily neurological folate deficiency syndrome in adults. Along with its inseparable 'testing partner' vitamin B12, neither is a plausible cause in my clinical practice for a symptomatic glove-and-stocking peripheral neuropathy (although B12 deficiency is a very important cause of dorsal column degeneration, which presents

Box 2 The clinical spectrum of neurological dysfunction in coeliac disease (from Hadfivassiliou et $a I^{4}$ )
Ataxia
Sensori-motor axonal neuropathy
Mononeuritis multiplex
Motor neuropathy
Small fibre neuropathy
Mixed axonal/demyelinating neuropathy
Myopathy
White matter changes on MRI with headache
Stiff-person syndrome
Neuromyotonia

differently). Serum folate can be, as in the case cited above, a surrogate for more generalised malabsorption, and undoubtedly levels should always be supplemented in pregnant women, especially those with epilepsy.

\section{Motor neurone disease}

The patient has a progressive motor syndrome with minimal sensory involvement. He has upper and lower motor neurone involvement in the same territories, and could as such be given a label of 'possible MND' according to the El Escorial criteria. ${ }^{5}$ One might expect to see EMG evidence of denervation, and the MRI brain may or may not show corticospinal tract hyperintensity, so this would not be contributory. A slowly progressive and very rare upper motor neurone-only variant of MND is primary lateralising sclerosis (PLS). It often presents with early loss of balance, later with slowly progressive spastic paraparesis, or corticobulbar dysfunction, initially. Atrophy of the primary motor cortex, unlike typical ALS, is often visible in established PLS on standard brain imaging. Overall, there are few credible mimics of MND in routine clinical practice, but may be considered in slowly progressive cases ${ }^{6}$ (box 3 ).

\section{Spinal xanthomatosis}

Spinal xanthomatosis - a variant of cerebrotendinous xanthomatosis-is a slowly progressive spinal cord syndrome. Cerebrotendinous xanthomatosis is an autosomal recessive metabolic disorder caused by an enzyme deficiency that results in accumulation of cholesterol and cholestanol in body tissues, including the central nervous system and tendons (hence the name). A case series of seven patients with spinal

Box 3 Potential mimics of slowly progressive motor neuron disease

\section{Lower motor neurone syndromes}

- Multifocal motor neuropathy: asymmetrical, typically affecting forearm extensors, causes weakness without wasting, no respiratory involvement.

- Inclusion body myositis: symmetrical, typically affecting quadriceps or medial forearms, CK often raised (not given in this case).

\section{Upper motor neurone syndromes}

- Hereditary spastic paraparesis: symmetrical paraplegia, can sometimes involve arms and dorsal columns, often a family history.

- Adrenomyeloneuropathy (X-linked adrenoleukodystrophy): symmetrical, slowly progressive paraparesis, often bladder involvement, very long chain fatty acids can be falsely negative. 
xanthomatosis indicated an age of onset in the $20 \mathrm{~s}$ and $30 \mathrm{~s}^{7}$ These patients had pyramidal and dorsal column signs at presentation, with most patients also having cataracts. The classic xanthomas and usual neurological symptoms (seizures, dysarthria, dementia, polyneuropathy and cerebellar symptoms) were often absent. MRI scanning showed white matter lesions in the cerebellum and spinal cord. Blood tests confirmed elevated serum cholestanol and/or cholesterol with increased urinary bile alcohols. This case series showed that serum cholesterol can be normal but cholestanol is always elevated.

\section{Demyelination}

Multiple sclerosis can present with hemiparesis and is a diagnosis worth considering, though I would expect sensory disturbance and bladder involvement. If this is multiple sclerosis, then I would favour primary progressive multiple sclerosis as the subtype.

\section{Syphilis}

One other point concerns syphilis: we are told this patient had 'negative syphilis serology', but venereal disease research laboratory (VDRL) can be negative in late syphilis. The FTA-Abs (fluorescent treponemal antibody absorption) test remains positive after primary infection, despite treatment (unlike VDRL). Overall, I do not think this diagnosis is tenable clinically.

\section{Copper deficiency}

Copper myeloneuropathy typically causes symmetrical neurological dysfunction with anaemia, so is unlikely to be the cause of this patient's symptoms (we are not given his copper levels). Cases have been generally confined to patients with zinc overload (commonly associated with excessive use of dental fixative ${ }^{8}$ ) or those with previous gastric surgery. ${ }^{9}$

\section{Cord compression}

A remaining option is cervical spinal cord compression. The lack of spino-thalamic tract sensory symptoms or sphincter involvement does not put me off. I recall a case known to my colleague, Professor Kevin Talbot, of a middle-aged man with a 6-month history of progressive weakness and wasting of both shoulder girdles, and deltoid fasciculation. His CK was elevated at 1486. EMG showed chronic partial denervation including his quadriceps, and a deltoid muscle biopsy was reported to show denervation. Clinically, this resembled the 'flail arm' slowly progressive variant of MND; ${ }^{10}$ however, the MRI showed cervical cord compression due to a posterior dural cystic expansion presumed due to a prior traumatic tear. His progression arrested without surgical intervention, and he has remained stable since.

\section{Mills' syndrome}

The Dean of American neurology, Charles Karsner Mills, described a slowly advancing hemiplegia, usually spreading from leg to arm, in 1900. In the few published cases subsequently grouped under this eponym, patients typically showed bilateral pyramidal signs, and sometimes also amyotrophy, but without fasciculation. The sensory examination was usually normal. The syndrome is sometimes considered a hemiplegic form of primary lateral sclerosis. If this patient had attended my clinic, I would have given him the clinical label of Mills' syndrome.

\section{Martin Turner's diagnosis}

I had hoped to be able to state unequivocally that this was a neurodegenerative form of Mills' syndrome, within the spectrum of MND. However, I think the diagnosis may be a structural lesion affecting the cervical cord, particularly, given the dorsal column involvement clinically. I am uncertain about the nature of this structural lesion, and it may even be developmental in origin given the time course.

\section{QUESTIONS FOR DR TURNER}

Q: Given the significant amount of weight loss, do you not think that a metabolic cause is the cause of his symptoms?

MT: It's possible, but I think this is much less likely given the asymmetry of the symptoms and signs.

Q: What about the role of amiodarone? We know that amiodarone has multiple effects on the thyroid; do you think this may have been an odd presentation of thyroid dysfunction?

MT: Again, I think it is difficult to explain the marked asymmetry with a systemic condition like amiodarone toxicity or thyroid dysfunction. The duration of his symptoms - 40 years - would also be very much against amiodarone toxicity.

Q: Do you think the sensory symptoms are relevant?

MT: I think these are 'red herrings'. The sensory symptoms suggest a right ulnar nerve neuropathy. The abnormalities on examination are rather non-specific.

Q: Are the bulbar symptoms important?

MT: This patient developed progressive dyspnoea, type 2 respiratory failure, and eventually died of aspiration pneumonia. These suggest that there was bulbar dysfunction. However, these features are common in many terminally ill patients, particularly in the frail elderly patient with heart failure.

\section{PATHOLOGY}

Post-mortem examination of the body identified bilateral bronchopneumonia, cardiomegaly with associated features of heart failure, and extensive pleural plaques in keeping with asbestosis exposure.

Macroscopic examination of the brain showed marked cortical atrophy predominantly affecting the frontal and parietal areas. The brainstem was normal. 
Microscopic examination showed small lacunar infarcts with associated small vessel disease, but no major involvement of the internal capsules. There was no evidence of asymmetric degeneration of the cerebral peduncles, corticospinal fibres in the pons, or pyramids. There was a normal population of Betz cells in the primary motor cortex.

Macroscopic inspection of the spinal cord showed a grey gelatinous appearance of the ventro-lateral cervical and upper thoracic spinal cord. Microscopic examination of the spinal cord showed loss of anterior horn cells, with small anterior nerve roots compared with the posterior nerve roots. There was vacuolation and gliosis present predominantly within the anterior and lateral aspects of the spinal cord, including the cortico-spinal tracts, although the dorsal columns were unaffected. The ventro-lateral cervical and upper thoracic cord lesions appeared to be long-standing, with evidence of scar tissue and downregulation of microglia, and no active inflammation.

Immunohistochemistry of the brain and spinal cord was, on the whole, negative for $\beta$-amyloid, tau, $\alpha$-synuclein, ubiquitin, nucleosporin p62, FUS (RNA binding protein FUS) or TDP-43 pathology. There were several ubiquitin-expressing neuronal inclusions within the 10th and 12th cranial nerve nuclei in the medulla, which may explain the swallowing difficulties. However, these ubiquitin-positive staining areas in the brainstem were negative for the ALS hallmark protein TDP-43. A myelin stain showed striking asymmetrical demyelination of the anterior and lateral spinal cord, including the cortico-spinal tracts (figure 1).

The appearances are unusual and may represent the pathological appearances of Mills' syndrome. The underlying pathophysiology remains uncertain.

\section{FINAL PATHOLOGICAL DIAGNOSIS}

Probable Mills' syndrome.

\section{POST-PATHOLOGY DISCUSSION}

Martin Zeidler (MZ): I'd like to highlight the importance of considering postmortem examination in patients with rare or unusual conditions. In this case, having come to the conclusion that this was a degenerative disorder conforming to the clinical description given by Mills, I had discussed the issue of postmortem with the patient. Doctors may be unwilling to discuss this issue with patients because of a presumption that the patient will be upset or distressed by the discussion. In reality, patients and their relatives are often willing to discuss postmortem examination and this should clearly be done before the patient becomes too unwell to have this conversation. In this case, my impression was that the patient was not distressed about the conversation we had, but I think it did upset his family initially.

Audience member: Could this be a chronic injury from electrocution? He was an electrical engineer who was right-handed.

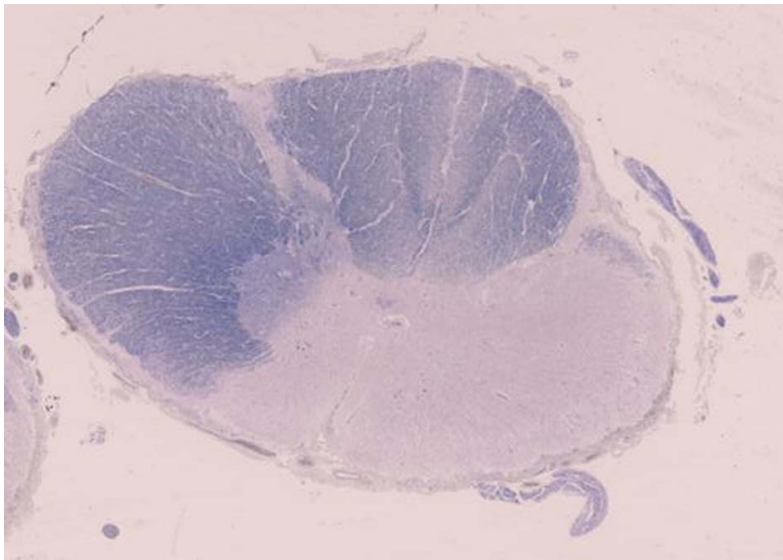

Figure 1 Transverse section of patient's spinal cord stained with Luxol Fast Blue, showing lack of myelin staining in ventral cord with preservation of dorsal cord myelin staining.

Colin Smith (CS): There were no specific features of the pathology to suggest electrocution.

MZ: There was no mention in the history of previous electrocution.

Audience member: What about a previous myelitis (of any cause)?

Martin Turner (MT): A clinically isolated syndrome of demyelination or other inflammatory process could certainly cause the clinical symptoms listed, but the history is of gradual onset with clear progression.

CS: There is no evidence of active myelitis found in the postmortem examination.

Audience member: Could this be a previous injury with gradual subsequent decompensation?

CS: Whilst possible, there is no evidence to support this from either history or pathology.

\section{MILLS' SYNDROME}

\section{Definition of Mills' syndrome}

There is no universally accepted definition of Mills' syndrome. The most widely accepted clinical definition is of a slowly progressive unilateral motor deficiency with unilateral or asymmetrical pyramidal signs but without sensory features. The presence of bulbar symptoms, sphincter disturbance, fasciculation or cognitive symptoms remains disputed. It is important to emphasise that this condition is a 'syndrome' (i.e., a set of associated symptoms and signs without an agreed underlying pathological basis) rather than a 'disease'. The rarity of the diagnosis (fewer than 50 cases reported in the literature since the original case in 1900) means that doubt remains as to the nosological validity of Mills' syndrome.

\section{The original case}

Charles Karsner Mills (1845-1930, figure 2) was a professor of neurology at the University of Pennsylvania. His eponymous syndrome was first presented to the Philadelphia Neurological Society in 
1899, before publication in $1900 .{ }^{11}$ Mills described a 52-year-old man with a 2-year history of slowly progressive right-sided weakness, affecting the leg initially before progressing to the arm, then face. There were no sensory or sphincter symptoms. Examination showed right-sided hemiatrophy and hemiparesis, with tone and reflexes increased on the affected side. Sensory examination was normal, as was the remainder of the neurological examination. The paper described two other similar cases, both with progressive hemiparesis but, unlike the index case, they also had sensory and sphincter symptoms. Of these two additional cases, one was lost to follow-up and the other was diagnosed with neurosyphilis. Several other cases, eight in total, were added in a case series published in $1906 .{ }^{12}$

\section{Subsequent case reports}

Two cases were described in 1994 by Gastaut and Bartolomei. ${ }^{13}$ One patient had an 18-year history of hemiparesis, starting at age 49 years, progressing from the right leg to right arm and latterly involving the bladder. Repeated investigations (including EMG,

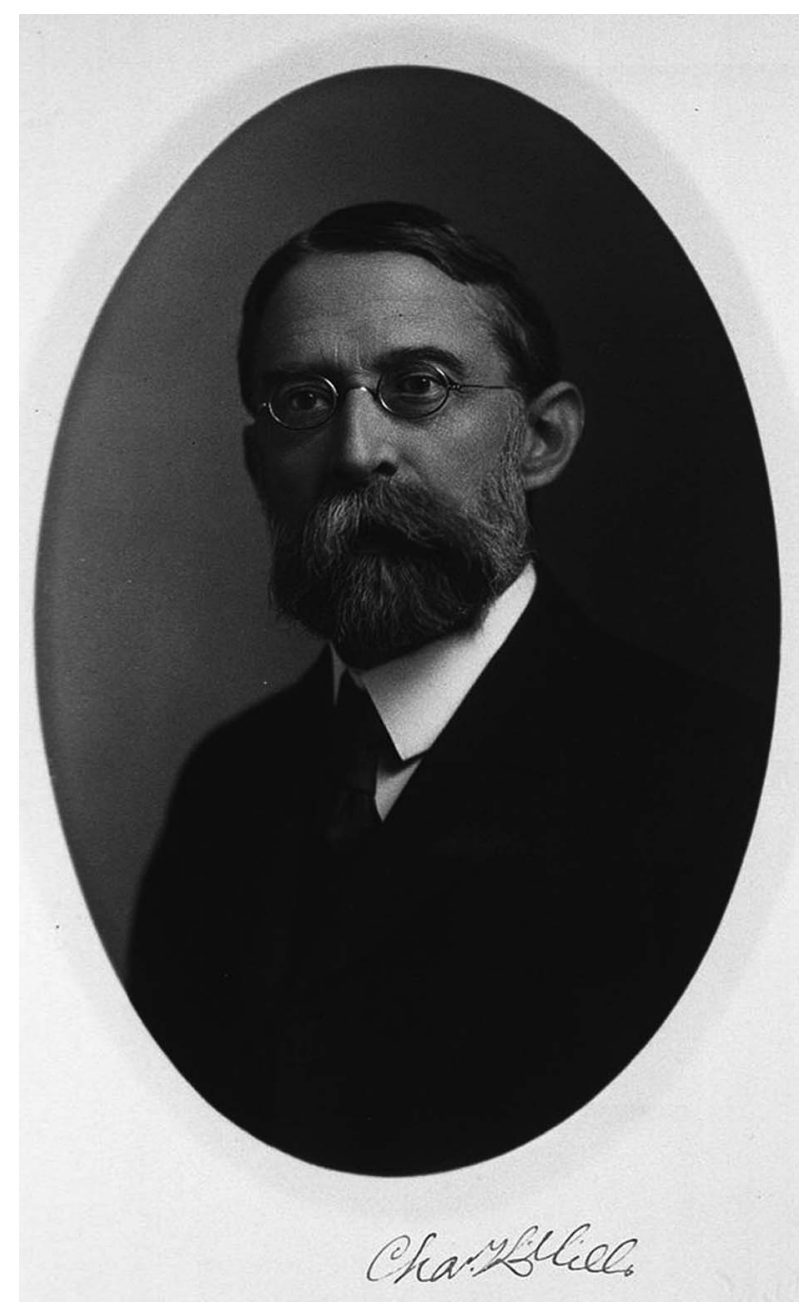

Figure 2 Charles Karsner Mills. electroencephalography, CSF analysis, brain CT, brain MRI, spine MRI, contrast myelography, syphilis serology, visual/auditory/somatosensory evoked potentials and spinal angiography) did not show any abnormalities except for a slightly raised CSF protein $(0.6 \mathrm{~g} / \mathrm{L})$. The second patient had a 17 -year history, starting aged 25 years. The hemiparesis began in the left hand before spreading to the left arm, leg and, finally, the left side of the face. Again, full investigations did not show any significant abnormalities. By 1994 , only 15 cases had been described in the scientific literature (box 4). It is important to recognise that the early cases are from an era without advanced investigations-including imaging-and may, therefore, include many cases that would not be diagnosed as Mills' syndrome today, such as brain tumours, cord compression or multiple sclerosis.

A French case series in 2007 of eight patients with suspected Mills' syndrome found a final diagnosis of Mills' syndrome in only three. ${ }^{14}$ The remainder had myelitis of unknown origin (two patients), primary lateral sclerosis, primary progressive multiple sclerosis or antiphospholipid syndrome.

There is one report of a patient with concurrent Mills' syndrome and Waldenström's macroglobulinaemia, ${ }^{15}$ although a causative link remains uncertain.

A patient with Mills' syndrome associated with aphasia has been reported. ${ }^{16}$ This patient had upper motor neurone weakness affecting the right arm, leg and face, together with marked difficulty generating speech. He went on to develop bulbar dysfunction and respiratory failure, dying within 2 years of symptom onset. A postmortem examination showed profound left hemisphere atrophy, including the primary motor cortex and temporal lobe. Histology found Bunina bodies (eosinophilic intraneuronal inclusions), which are a specific pathological marker of ALS. Microglial activation was noted in the right lateral cortico-spinal tract but also contralateral uncrossed anterior fibres within the spinal cord.

Box 4 Features of Mills' syndrome in 15 cases (from Gastaut 1994 ${ }^{13}$ )

Unilateral pyramidal signs: 8/15 (remainder had bilateral, usually asymmetrical, signs)

- Amyotrophy without fasciculation: 6/15 (remainder did not have amyotrophy)

- Facial involvement: $5 / 15$

- Sensory disturbance: $3 / 15$ (minimal changes only)

- Ascending symptoms: $13 / 15$

- Bilateral symptoms: 5/15 (only in very advanced disease)

- Family history: 0/15 
However, the case is not typical of Mills' syndrome given the rapid progression of symptoms.

\section{Microglial activation}

Cerebral microglial activation is increased in patients with ALS, as shown by in vivo positron emission tomography (PET) studies. ${ }^{17}$ Two patients with clinical features similar to those described by Mills had PET scanning with a specific ligand for microglial activation. ${ }^{18}$ The first patient had a 20 -year history of rightsided upper motor neuron symptoms and signs. There was evidence of increased cerebral microglial activation in the superior frontal lobe and the supplementary motor area in the left hemisphere. The second patient had a 3-year history of left-sided pyramidal weakness with an inflammatory lesion at C4 on MRI cervical cord. In this patient, PET scanning was normal, although the cervical cord was not imaged.

\section{Aetiology}

Mills listed five possible causes for his patient's symptoms and signs (box 5) but stated that the case did not "seem to fit in exactly with any one of these diagnoses". Subsequent speculation about the pathophysiology of Mills' syndrome has largely been confined to these five possible aetiologies, discussed in more detail below.

Solitary or multiple sclerosis

Unilateral disseminated sclerosis was discussed by Mills, but thought less likely given the absence of the 'ordinary train of symptoms' usually found in this condition.

A case series was published of seven patients with so-called 'solitary sclerosis'. ${ }^{19}$ These patients had progressive symptoms secondary to a single demyelinating lesion in the brainstem or upper cervical cord. Over the 6-year evaluation period, none developed new MRI lesions and none had relapses affecting other areas of the nervous system. Of the seven patients, two had progressive hemiparesis analogous to Mills' syndrome. However, both patients had sensory and bladder symptoms, unlike the usual descriptions of patients with Mills' syndrome. Additionally, one patient had positive oligoclonal bands in the CSF.
Unilateral ALS or primary lateral sclerosis

Mills thought that unilateral ALS was the most likely diagnosis and referred to a previous series of seven patients with this diagnosis. However, he felt that the lack of spasticity and contractures counted against this diagnosis, although suggested that these features may develop later.

Gastaut stated that Mills' syndrome might be considered as a hemiplegic form of PLS ${ }^{13}$ and most subsequent commentators seem to agree with this viewpoint. Whereas the pathological findings of ALS and the overlap disorder of frontotemporal lobar degeneration (FTLD) are well documented, the pathological changes of PLS are poorly described. In sporadic ALS, the typical pathological findings include neuronal cytoplasmic inclusions positive for ubiquitin and TDP-43 (TAR DNA binding protein 43). ${ }^{20}$ By contrast, Pringle et $a l^{21}$ described a case of PLS (without FTLD) with prominent atrophy of both precentral gyri and absence of Betz cells (pyramidal cells longer than $50 \mu \mathrm{m}$ ) in layer five of the cortex of the precentral gyri, with a reduction in the amount of pyramid cells in layers 3 and 5. No intraneuronal inclusions were noted, but the report precedes the discovery of TDP-43 as a specific marker of ALS/FTLD. Josephs and Dickson ${ }^{22}$ identified two cases of PLS associated with FTLD. In these cases, the pathology showed fronto-temporal neuronal loss, with ubiquitin and TDP-43-positive inclusions in these areas. Examination of the motor cortices showed neuronal loss, gliosis and reduction of Betz cells-but no TDP-43 immunoreactivity.

Focal cerebral lesion

The third possibility, a slowly evolving focal cerebral lesion, was thought unlikely by Mills given the specificity and progression of the motor symptoms, as well as the lack of 'Jacksonian epilepsy'.

There is a case report of a 69-year-old man presenting with a slowly progressive left leg weakness, associated with unilateral atrophy and asymmetrical reflexes. ${ }^{23}$ His symptoms progressed and spread proximally to involve the left arm as well. An MR scan of brain showed multiple lacunar infarcts affecting the right hemisphere, and the eventual diagnosis was of a secondary Mills' syndrome-that is, Mills' syndrome caused by multiple unilateral cerebral infarcts.

Motor neuropathy

Mills dismissed a degenerative motor neuritis (peripheral neuropathy) as the cause of his patient's condition, given the unilateral symptoms and asymmetry of the tendon reflexes.

\section{Functional hemiparesis}

Finally, a functional hemiparesis was thought possible but improbable by Mills, given the persistence and progression of symptoms in the absence of 'hysterical 
stigmata'. The presence of unequivocal signs in our case and others also excludes this.

\section{Pathology}

There are three reports of postmortem studies in patients thought to have Mills' syndrome, although none of these cases fully fit the definition of this disorder.

The first report was referenced by Mills in his original paper and showed several plaques of sclerosis, with the most extensive plaque affecting the right side of the cord only. There was a suggestion that some of these plaques were reminiscent of neuro-syphilitic lesions; the patient had an 11-year history of syphilis at the time of death.

The second postmortem report was published by Mills in $1903 .{ }^{24}$ This case is of a 60 -year-old man who had a 13-year history of progressive weakness, affecting the right leg, then right arm and finally left leg (ie, triplegia, rather than the hemiplegia characteristically described in Mills' syndrome). The reflexes were brisker in the affected limbs but there were no sensory symptoms. Pathological examination of the brain and spinal cord showed distinct "degeneration of the right crossed and left direct pyramidal tracts", with the contralateral pathways being "much less degenerated". The right pyramidal changes extended into the medulla oblongata and pons, which again were much more affected than the left pyramidal tracts. The anterior horn cells were reported as being normal, having "the usual appearance in persons of the same age". The left frontal lobe contained Betz cells, reported as normal in appearance.

The third postmortem study has already been discussed: a case of very rapidly progressive unilateral upper motor neuron weakness with aphasia, developed bulbar dysfunction and died of respiratory failure. This case is atypical of Mills' syndrome given the rapid progression and presence of aphasia.

Our case is, therefore, the first modern description of the pathological findings in typical Mills' syndrome.

\section{FINAL COMMENT FROM DR TURNER}

I was referred another Mills' syndrome case by a UK colleague recently. Review of the MRI scans showed an appropriately lateralised brainstem white matter lesion. I thought the most likely explanation in that case was progressive myelopathy from a solitary demyelinating lesion given the Mayo Clinic case series published by Schmalstieg et al. ${ }^{19}$ In this case, in light of the pathology, I think a solitary demyelinating lesion is also plausible, with age-related decompensation being responsible for the slow progression over time.

Acknowledgements We are grateful to the patient's family for allowing us to discuss and publish this case.
Contributors $\mathrm{RD}$ and $\mathrm{MZ}$ contributed towards writing the article. CS provided all pathology material and contributed towards writing the article. MRT critically appraised the case and contributed towards writing the article. PMF undertook literature review on Mills' syndrome and contributed towards writing the article.

Competing interests None.

Patient consent Obtained.

Provenance and peer review Not commissioned; externally peer reviewed. This paper was reviewed by Andrew Chancellor, Tauranga, New Zealand.

\section{REFERENCES}

1 Turner MR, Talbot K. Functional vitamin B12 deficiency. Pract Neurol 2009;9:37-41.

2 Turner MR, Chohan G, Quaghebeur G, et al. A case of celiac disease mimicking amyotrophic lateral sclerosis. Nat Clin Pract Neurol 2007;3:581-4.

3 Ellis CM, Simmons A, Dawson JM, et al. Distinct hyperintense MRI signal changes in the corticospinal tracts of a patient with motor neurone disease. Amyotroph Lateral Scler Other Motor Neurone Disord 1999;1:41-4.

4 Hadjivassiliou M, Grunewald RA, Davies-Jones GA. Gluten sensitivity as a neurological illness. J Neurol Neurosurg Psychiatry 2002;72:560-3.

5 Brooks BR, Miller RG, Swash M, et al. El Escorial revisited: revised criteria for the diagnosis of amyotrophic lateral sclerosis. Amyotroph Lateral Scler Other Motor Neurone Disord 2002;1:293-9.

6 Turner MR, Talbot K. Mimics and chameleons in motor neurone disease. Pract Neurol 2013;13:153-64.

7 Verrips A, Nijeholt GJ, Barkhof F, et al. Spinal xanthomatosis: a variant of cerebrotendinous xanthomatosis. Brain 1999;122:1589-95.

8 Nations SP, Boyer PJ, Love LA, et al. Denture cream: an unusual source of excess zinc, leading to hypocupremia and neurologic disease. Neurology 2008;71:639-43.

9 Pal S, Malhotra RM, Davenport RJ, et al. Progressive unsteadiness in a 68-year-old man with longstanding abdominal pain and altered bowel habit. Pract Neurol 2009;9:210-20.

10 Hu MT, Ellis CM, Al-Chalabi A, et al. Flail arm syndrome: a distinctive variant of amyotrophic lateral sclerosis. J Neurol Neurosurg Psychiatry 1998;65:950-1.

11 Mills CK. A case of unilateral progressive ascending paralysis, probably representing a new form of degenerative disease. J Nerve Ment Dis 1900;27:195-200.

12 Mills CK. Unilateral ascending paralysis and unilateral descending paralysis. JAMA 1906;47:1638-45.

13 Gastaut JL, Bartolomei F. Mills' syndrome: ascending (or descending) progressive hemiplegia: a hemiplegic form of primary lateral sclerosis? J Neurol Neurosurg Psychiatry 1994;57:1280-1.

14 Lachaud S, Soriani MH, Delmont E, et al. Mills' syndrome: a rare clinical entity. Rev Neurol (Paris) 2007;163:335-40.

15 Rajabally YA, Hbahbih M, Abbott RJ. Hemiplegic ALS: Mills syndrome. Neurology 2005;64:1984-5.

16 Baumer D, Butterworth R, Menke RA, et al. Progressive hemiparesis (Mills syndrome) with aphasia in amyotrophic lateral sclerosis. Neurology 2014;82:457-8.

17 Turner MR, Cagnin A, Turkheimer FE, et al. Evidence of widespread microglial activation in amyotrophic lateral 
sclerosis: an ${ }^{11} \mathrm{C}-(\mathrm{R})-\mathrm{PK} 11195$ positron emission tomography study. Neurobiol Dis 2004;15:601-9.

18 Turner MR, Gerhard A, Al-Chalabi A, et al. Mills' and other isolated upper motor neurone syndromes: in vivo study with ${ }^{11} \mathrm{C}-(\mathrm{R})-\mathrm{PK} 11195$ PET. J Neurol Neurosurg Psychiatry 2005;76:871-4.

19 Schmalstieg WF, Keegan BM, Weinshenker BG. Solitary sclerosis: progressive myelopathy from solitary demyelinating lesion. Neurology 2012;78:540-4.

20 Dickson DW, Josephs KA, Amador-Ortiz C. TDP-43 in differential diagnosis of motor neurone disorders. Acta Neuropathol 2007;114:71-9.
21 Pringle CE, Hudson AJ, Munoz DG, et al. Primary lateral sclerosis: clinical features, neuropathology, and diagnostic criteria. Brain 1992;115:495-520.

22 Josephs KA, Dickson DW. Frontotemporal lobar degeneration with upper motor neurone disease/primary lateral sclerosis. Neurology 2007;69:1800-1.

23 Dalla Volta G, Magoni M, Vangi D, et al. Role of MRI in the diagnosis of Mills syndrome. Ital J Neurol Sci 1989;10:519-21.

24 Mills CK, Spiller WG. A case of progressively developing hemiplegia, later becoming triplegia, resulting from primary degeneration of the pyramidal tracts. J Nerve Ment Dis 1903;30:385-97. 